\title{
BMJ Open Evolution of the burden of active hepatitis $C$ virus infection in England from September 2015 to September 2016: a repeated cross-sectional analysis
}

\author{
Laure Lacoin, ${ }^{1}$ Michael Hurst, ${ }^{2}$ Nathan R Hill, ${ }^{1,3}$ Jason Gordon, ${ }^{2}$ \\ Anna Maria Geretti, ${ }^{4}$ Richard Aspinall, ${ }^{5}$ Lynsey Corless, ${ }^{6}$ Yuxiang Gao-Du, ${ }^{6}$ \\ Liam Mistry, ${ }^{7}$ David Mutimer ${ }^{7}$
}

To cite: Lacoin L, Hurst M, Hill NR, et al. Evolution of the burden of active hepatitis $\mathrm{C}$ virus infection in England from September 2015 to September 2016: a repeated crosssectional analysis. BMJ Open 2019;9:e029066. doi:10.1136/ bmjopen-2019-029066

- Prepublication history and additional material for this paper are available online. To view these files, please visit the journal online (http://dx.doi org/10.1136/bmjopen-2019029066).

Received 10 January 2019 Revised 22 May 2019 Accepted 21 June 2019
Check for updates

(C) Author(s) (or their employer(s)) 2019. Re-use permitted under CC BY-NC. No commercial re-use. See rights and permissions. Published by BMJ.

For numbered affiliations see end of article.

Correspondence to Professor David Mutimer; david.mutimer@uhb.nhs.uk

\section{ABSTRACT}

Objective To evaluate the impact of treatment with new direct-acting antivirals (DAAs) on the prevalent hepatitis $\mathrm{C}$ virus (HCV) population in England.

Design A repeated cross-sectional analysis.

Setting Four secondary care hospitals in England. Participants Patients who, in 2015 and/or 2016, had chronic HCV infection and were alive were eligible, regardless of the type of $\mathrm{HCV}$ intervention received. Outcome measures Data including intravenous drug use (IVDU) status, HCV genotype, cirrhosis status, HCV treatment history, vital status and treatment outcomes were collected at two time points in 2015 and 2016 using electronic case report forms.

Results There were 1605 and 1355 patients with active chronic HCV in 2015 and 2016, respectively. Between 2015 and 2016, the proportion of patients with current IVDU increased ( $10.3 \%$ vs $14.5 \%$, respectively), while that of patients with cirrhosis $(28.2 \%$ vs $22.4 \%)$ and treatmentexperienced patients ( $31.2 \%$ vs $27.1 \%$ ) decreased. Among patients whose treatment outcome was known by 2016 , high cure rates were observed, with an overall sustained virological response rate of $93.2 \%$. From 2015 to 2016 , there was a progressive increase in the proportion of treated patients who were non-cirrhotic, with current IVDU and non-liver transplant recipients.

Conclusions The characteristics of patients with HCV remaining in contact with specialised care evolved with a changing landscape of treatment and related health policy. With increasing access to DAAs in UK, high cure rates were achieved in the study cohort.

\section{INTRODUCTION}

Worldwide, infection with the bloodborne hepatitis $\mathrm{C}$ virus (HCV) is estimated to cause 399000 deaths every year. ${ }^{1}$ Although some of those infected with HCV clear the virus spontaneously, the remaining 60\%-80\% develop chronic hepatitis C. ${ }^{1}$ Patients with chronic hepatitis are at high risk of developing severe liver disease, including cirrhosis (15\%-30\% risk over 20 years ${ }^{1}$ ) and hepatocellular carcinoma $\left(1 \%-3 \%\right.$ risk over 30 years $\left.^{2}\right)$, which
Strengths and limitations of this study

- The study describes how the characteristics of patients with hepatitis $\mathrm{C}$ virus (HCV) remaining in contact with specialised care evolved with the changing landscape of treatment since the introduction of direct-acting antivirals and provides important real-world data on the outcomes of antiviral treatment among a diverse patient cohort.

- Patient information was collected from four hospitals in England, and so the generalisability of our findings should be considered against the study setting and the range of data collected.

- The collection of patient information from four specialist hospitals gives valuable insight into the treatment of HCV in secondary care.

- The developments in the treatment of HCV in the last 2 years may mean that the characteristics of patients currently receiving treatment have changed from those described in our study.

- The retrospective nature of data collection could subject it to issues related to data access, completeness and accuracy.

directly cause the majority of HCV-related deaths. ${ }^{1}$ Consequently, chronic HCV is considered a major public health threat, ${ }^{34}$ affecting $214000^{3}$ people in the UK and 160000 in England alone. ${ }^{4}$ Intravenous drug use (IVDU) is a significant contributor to $\mathrm{HCV}$ spread; in 2016, more than half of the people in England who injected psychoactive drugs were also infected with $\mathrm{HCV}^{4}$

The recent availability of direct-acting antivirals (DAAs) offers many HCV-infected patients the chance of a cure, with sustained virological response (SVR) rates commonly exceeding $90 \%$ in clinical trials ${ }^{5-7}$ and realworld studies alike. ${ }^{8-12}$ While treatment recommendations are genotype (GT)-specific, ${ }^{13}$ new DAAs are generally considered to be safe and highly effective. In the UK, access 
to DAAs has been initially prioritised for patients with the most severe liver disease. This reflected the relatively high acquisition costs of DAAs compared with historical standards of care, and the significant patient and health system savings expected from the avoidance of HCV-related complications and mortality. ${ }^{14}$ Understanding the nature of the remaining untreated or uncured HCV population, and the outcomes of patients treated with DAAs, is important to both clinicians and policy makers in guiding ongoing and future efforts to eradicate $\mathrm{HCV}$ infection.

This study aimed to demonstrate the impact of new DAAs on the prevalent population with known HCV infection in England following their launch, facilitating the understanding of both the remaining burden of known HCV infection and the future needs of HCV-infected populations, including potential barriers to eradicating HCV. Specifically, the study aimed to address four objectives: (1) evaluate the change in size and characteristics of the population with active chronic HCV infection between September 2015 and September 2016 at four hospitals in England; (2) describe the antiviral treatment patterns and outcomes in adult patients with chronic HCV infection over a 1-year period from September 2015; (3) characterise patients who were lost to follow-up (LTFU), to understand how they may be re-engaged with care and antiviral treatment; and (4) provide insights into the likely emerging and future needs of patients with HCV in the DAA era, by describing the characteristics of patients who were newly referred during the 12-month observation period (ie, between 15 September 2015 and 15 September 2016) and comparing them with those of patients already in contact with care at study onset.

\section{METHODS}

\section{Patient and public involvement}

The study was retrospective and observational in design, and as such patients and the public were not involved in the design and implementation of the study.

\section{Study settings}

Within England, prior to June 2015, DAA usage was initially provided via early access schemes. Since June 2015, DAAs have been available, but the roll-out has been prioritised for people with advanced HCV-related liver disease, specifically patients with compensated cirrhosis extending to all GT1 patients and GT3 patients who were contraindicated to interferon (IFN)-based regimens. ${ }^{15}$ Although patients with cirrhosis were still prioritised, there was a further roll-out to non-cirrhotic patients from April 2016.

Within England, recent estimates put the undiagnosed HCV population at approximately 50\%. ${ }^{16}$ Testing for $\mathrm{HCV}$ is typically performed on a targeted basis within primary care, be it within general practice of specialised sexual health clinics based on high-risk groups, including but not limited to IVDU/other drug use, existing blood/ organ transplants (prior to 1992), originating from highrisk countries, in regular sexual contact with patients diagnosed with HCV and babies born to mothers diagnosed with HCV. ${ }^{17}$ Diagnoses are performed in primary care, and those positive will be referred to an HCV specialist within secondary care where treatment and monitoring take place.

\section{Study design}

This repeated open cohort study was based on historical data collection at two different time points: 15 September 2015 and 15 September 2016 (hereby referred to as 2015 and 2016, respectively), which served, respectively, as index dates for the 2015 and 2016 cohorts of patients with active HCV infection.

Four hospitals in England participated: Birmingham (Queen Elizabeth Hospital, Birmingham), Liverpool (Royal Liverpool University Hospital), Hull (Hull and East Yorkshire Hospitals NHS Trust) and Portsmouth (Queen Alexandra Hospital, Portsmouth). These hospitals were chosen to be broadly representative (geographical and sociodemographics) of patients in specialist care in England.

\section{Study population}

Study populations were defined with the four study objectives in mind. To evaluate the change in size and characteristics of the population with active chronic HCV infection between 2015 and 2016, two patient cohorts were created: 2015 active HCV cohort and 2016 active HCV cohort. The 2015 active HCV cohort included all alive adult patients with chronic HCV infection prior to 15 September 2015, defined as patients with viraemia at the most recent attendance at a specialist HCV appointment for any reason, patients who were on antiviral therapy, or patients who had recently completed antiviral therapy but were yet to complete the 12-week post-treatment assessment of response. The 2016 active HCV cohort included patients part of the 2015 active HCV cohort who were alive but not cured as of 15 September 2016, as well as newly referred patients post 15 September 2015 meeting the same inclusion criteria. Patients with a known diagnosis of HCV who had attended the study centres at some stage but who had not been seen within the 24-month period prior to the index date were excluded. Patients' demographic and clinical characteristics, and previous and current HCV treatment received at time of their last visit prior to the index date, were collected.

To describe antiviral treatment patterns and outcomes over 1 year from the index date in adult patients with chronic HCV infection, clinical characteristics, treatments received and vital status at the time of the last visit prior to the 2016 index date were collected for all patients included in the 2015 active HCV cohort, except for those without a visit in the preceding 24 months.

Patients who were included in the 2015 active HCV cohort but were not seen in the study centres within 24 months prior to the 2016 active HCV cohort index date 
were considered LTFU. Characterising this cohort was of interest, as it could help design strategies aiming to re-engage them with care and facilitate uptake of antiviral treatment among this potentially challenging patient group.

Finally, to predict the changing needs of patients with HCV enrolling for treatment in the DAA era, we characterised (as of 2016) eligible patients who were newly referred to the study centres within the 1-year observation period and compared them with the prevalent 2015 active HCV cohort, which included patients who had been in contact with secondary care services over a period prior to DAA introduction.

\section{Data collection}

An electronic case report form (eCRF), designed to capture demographic and clinical data collected from existing databases or historical review of medical records, was used as the primary instrument for data collection by hospital staff members, which was entirely retrospective and performed poststudy.

Data collected within the eCRF at each time point included the following: IVDU status, HCV genotype; most recent viral load; liver fibrosis status (non-cirrhotic (F0-F2, F3, no F-score), cirrhotic (compensated, decompensated, without precision)); HIV coinfection; liver transplantation status; previous and current HCV treatment received (regimens received and start date/end date); and vital status (alive, death related to HCV infection, death for other reason). Furthermore, outcomes related to treatment received during the observation period were recorded, including no response, breakthrough, interruption due to adverse drug reaction, relapse, SVR achieved and unknown.

\section{Statistical analyses}

Patient demographic and clinical characteristics were described using summary statistics. Continuous variables were reported as mean with SD, median, minimum, maximum, and first and third quartiles. Categorical variables were reported as counts, proportions and its 95\% CI. The number and percentage of patients with missing data for each variable were described. Due to the retrospective nature of the trial, missing data were categorised as missing for all continuous variables. The changes in the size and the characteristics of the population with active infection between 2015 and 2016 were described by calculating the absolute difference in patients counts and variables distribution (\%) between 2015 and 2016.

\section{RESULTS}

The patient populations are summarised in figure 1 . The 2015 active HCV cohort included 1605 patients with active HCV infection at index date. Of those, $22.9 \%$ achieved SVR prior to 2016 (368 patients, including 3 patients who died after achieving SVR) and $1.6 \%$ died without achieving SVR (26 patients). Among the 2015 active HCV cohort, $154(9.6 \%)$ patients with no visits within the last 24 months prior to 2016 were considered LTFU and were excluded from the 2016 active HCV cohort.

An additional 337 patients with HCV were newly referred to the participating centres during the 12-month observation period, of whom $88.4 \%$ had an active HCV infection at the time of the last visit before 2016, 10.1\% achieved SVR after referral and before 2016, and 1.5\% died without SVR. Thus, the 2016 active HCV cohort included 1355 patients with active HCV infection at index date.

\section{Change in the characteristics of the active HCV population between 2015 and 2016}

Overall, the active HCV population reduced by 250 patients $(-15.6 \%)$ between 2015 and 2016 (table 1). The largest proportion of patients from both 2015 and 2016 active HCV cohorts were enrolled at Birmingham (701 and 459 patients, respectively). The changes in the size of the active HCV population varied widely across

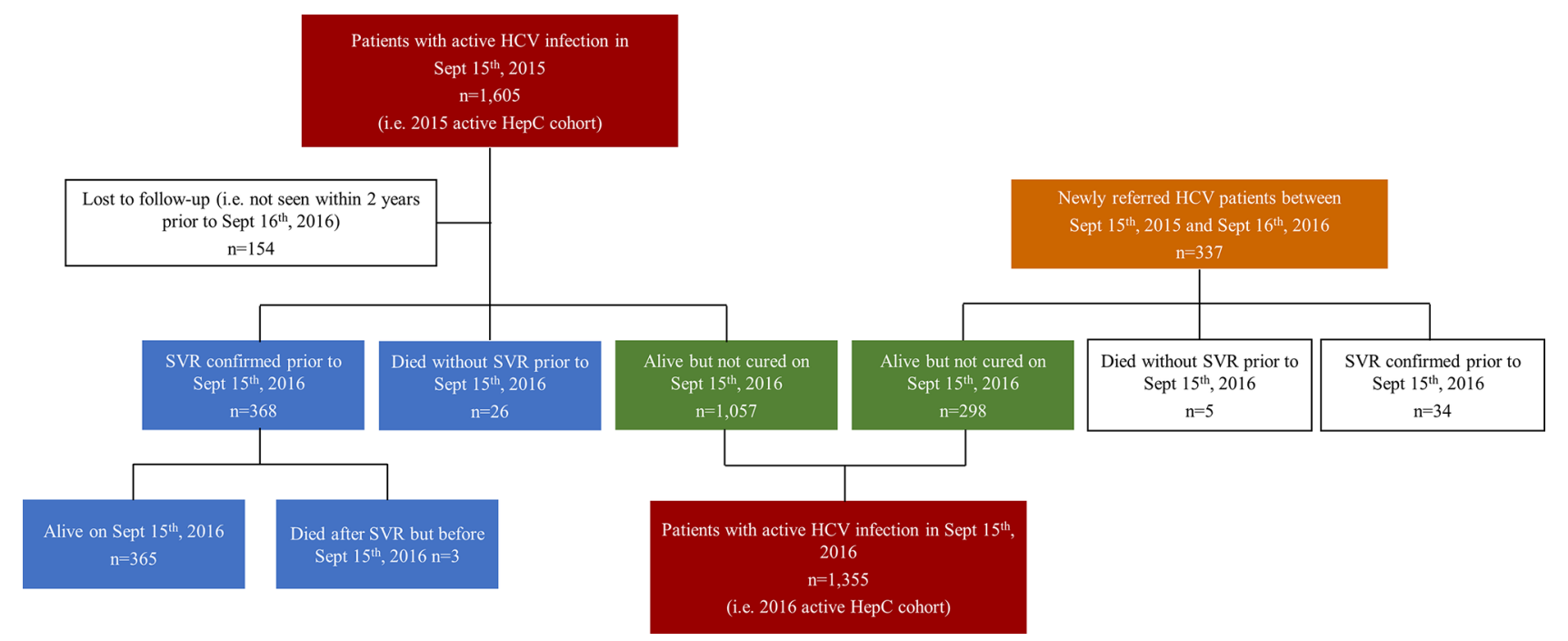

Figure 1 Study patient populations. HCV, hepatitis C virus; HepC, hepatitis C; SVR, sustained virological response. 
Table 1 Change in the characteristics of the active HCV population between 2015 and 2016

2015 active HCV cohort

$\% \quad(95 \% \mathrm{Cl})$

Overall population

Centre

\begin{tabular}{|c|c|c|c|c|c|c|c|c|}
\hline Birmingham & 701 & 43.7 & (41.25 to 46.1$)$ & 459 & 33.9 & (31.4 to 36.4 ) & -242 & -9.8 \\
\hline Hull & 307 & 19.1 & (17.20 to 21.1$)$ & 401 & 29.6 & (27.2 to 32.0 ) & 94 & 10.5 \\
\hline Liverpool & 485 & 30.2 & (27.97 to 32.5 ) & 410 & 30.3 & (27.8 to 32.7 ) & -75 & 0.0 \\
\hline \multicolumn{9}{|l|}{$\begin{array}{l}\text { Age at index date } \\
\text { (years) }\end{array}$} \\
\hline Q1-Q3 & \multicolumn{2}{|l|}{$41-56$} & & \multicolumn{2}{|l|}{$40-55$} & & & \\
\hline Minimum-maximum & \multicolumn{2}{|l|}{$18-83$} & & \multicolumn{2}{|l|}{$19-84$} & & & \\
\hline Male & 1197 & 74.6 & (72.4 to 76.7 ) & 997 & 73.6 & (71.2 to 75.9 ) & -200 & -1.0 \\
\hline \multicolumn{9}{|l|}{ IVDU } \\
\hline Not recorded & 429 & 26.7 & (24.6 to 28.9 ) & 283 & 20.9 & (18.7 to 23.1$)$ & -146 & -5.8 \\
\hline \multicolumn{9}{|l|}{ Genotype } \\
\hline GT1 & 704 & 43.9 & (41.4 to 46.3 ) & 582 & 43.0 & (40.3 to 45.6 ) & -122 & -0.9 \\
\hline GT2 & 72 & 4.5 & (3.5 to 5.5$)$ & 60 & 4.4 & (3.3 to 5.5$)$ & -12 & -0.1 \\
\hline GT3 & 660 & 41.1 & (38.7 to 43.5 ) & 556 & 41.0 & (38.4 to 43.7 ) & -104 & -0.1 \\
\hline Other & 106 & 6.6 & (5.4 to 7.8$)$ & 91 & 6.7 & (5.4 to 8.0$)$ & -15 & 0.1 \\
\hline Missing information & 63 & 3.9 & (3.0 to 4.9 ) & 66 & 4.9 & (3.7 to 6.0 ) & 3 & 0.9 \\
\hline \multicolumn{9}{|l|}{ Liver disease status } \\
\hline No cirrhosis & 1060 & 66.0 & (63.5 to 68.6) & 997 & 73.6 & (71.2 to 75.9$)$ & -63 & 7.5 \\
\hline Non-cirrhotic, F3 & 154 & 9.6 & (8.0 to 11.2$)$ & 137 & 10.1 & (8.5 to 11.7$)$ & -17 & 0.5 \\
\hline Non-cirrhotic, F0-F2 & 624 & 38.9 & (36.3 to 41.5$)$ & 505 & 37.3 & (34.7 to 39.8$)$ & -119 & -1.6 \\
\hline $\begin{array}{l}\text { Non-cirrhotic, no } \\
\text { score }\end{array}$ & 282 & 17.6 & (15.5 to 19.6$)$ & 355 & 26.2 & (23.9 to 28.5 ) & 73 & 8.6 \\
\hline Missing information & 93 & 5.8 & (4.6 to 7.0$)$ & 54 & 4.0 & (2.9 to 5.0$)$ & -39 & -1.8 \\
\hline \multicolumn{9}{|l|}{ Child-Pugh score* } \\
\hline A & 323 & 71.5 & (69.1 to 73.9$)$ & 199 & 65.5 & (60.1 to 70.8$)$ & -124 & -6.0 \\
\hline B & 55 & 12.2 & (10.4 to 13.9 ) & 43 & 14.1 & (10.2 to 18.06$)$ & -12 & 2.0 \\
\hline C & 13 & 2.9 & (2.0 to 3.8 ) & 13 & 4.3 & (0.0 to 6.6$)$ & 0 & 1.4 \\
\hline Missing information & 61 & 13.5 & (11.7 to 15.3 ) & 49 & 16.1 & (12.0 to 20.3 ) & -12 & 2.6 \\
\hline
\end{tabular}
and 2016

2016 active HCV cohort

Difference Difference (n)

(\%)

$\begin{array}{llll}\mathbf{n} & \% & \mathbf{( 9 5 \%} \mathbf{C l}) & \text { (n) } \\ 1355 & & & -250\end{array}$

1605

1355

250

Changes in active HCV cohort between 2015 


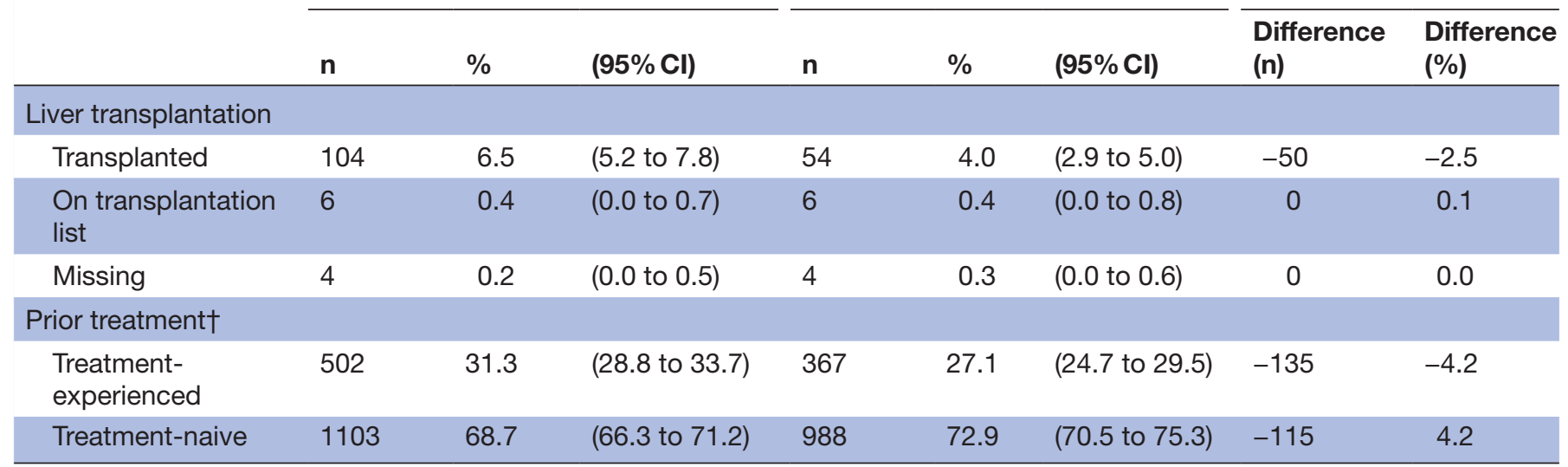

Continuous variables represented as mean. Categorical variables denoted as \% $(95 \% \mathrm{Cl})$.

*Includes only patients with cirrhosis.

†Does not consider patients currently on treatment.

F0-F2, fibrosis stage 0-2; F3, fibrosis stage 3; GT1, genotype 1; GT2, genotype 2; GT3, genotype 3; HCV, hepatitis C virus; IVDU, intravenous drug use.

the different centres. The number of patients enrolled with active HCV infection decreased between 2015 and 2016 at Birmingham, Liverpool and Portsmouth, the more important decrease being observed at Birmingham (from 701 patients to 459 patients between 2015 and 2016). Conversely, Hull experienced a substantial rise in the number of patients, increasing its enrolment by 94 patients during the observation period $(19.1 \%$ of total active HCV cohort in 2015 and $29.6 \%$ in 2016). These variations are likely to be related to the difference in the characteristics of the population covered by the different centres (online supplementary tables ST1ad); notably, about a third of patients with active HCV enrolled at Birmingham, Liverpool and Portsmouth in 2015 had cirrhosis $(32.4 \%, 33.2 \%$ and $38.4 \%$, respectively), compared with just $6.8 \%$ at Hull. Furthermore, the 2015 active HCV cohort at Birmingham, Liverpool and Portsmouth included few patients with current IVDU $(2.1 \%, 10.3 \%$ and $7.1 \%$, respectively); conversely, at Hull, this proportion was as high as $30.0 \%$. Patients enrolled at Hull were also considerably younger than in the other three hospitals (41.6 years vs 48.3-53.2 years). Discrepancies between centres are likely attributable to the target population, as Birmingham is a tertiary referral centre for complex/advanced liver disease including transplantation, whereas Hull serves a large prison population.

Regarding the changes in the characteristics of the active HCV population across all four study centres (table 1 and online supplementary tables STla-d), no substantial differences between the two cohorts were observed in terms of age and genotype distribution. However, a $4.2 \%$ increase in the proportion of patients with current IVDU was observed, primarily attributable to the increased number of patients enrolled at Hull within the 2016 active HCV cohort. As mentioned above, IVDU was far more common among patients enrolled at this centre due to the volatility of the prison population, affecting $27.7 \%$ of patients in 2016 and $30.0 \%$ in 2015 .

At the same time, a decrease in the proportion of patients with cirrhosis from $28.2 \%$ to $22.4 \%$ was observed, consistent with these patients being prioritised for treatment. The reduction in patients with cirrhosis was observed in patients enrolled at Birmingham, Liverpool and Portsmouth but not at Hull (online supplementary tables STla-d). Similarly, the proportion of transplanted patients (from $6.5 \%$ to $4.0 \%$ ) and patients who are treatment-experienced (including both IFN-based and non-IFN-based regimens) also decreased (from $31.2 \%$ to $27.1 \%$ ) between 2015 and 2016. Generally, similar trends were observed between 2015 and 2016 active HCV cohorts when stratifying by GT1 and GT3 (online supplementary tables ST1e and ST1f, respectively). However, fewer post-transplant patients with active GT3 HCV infection were treated and removed from the cohort compared with GT1-infected patients, so that the number of post-transplant patients with active GT1 HCV decreased from 48 in 2015 to 15 in 2016 (online supplementary table ST1e), while the corresponding decrease for GT3 infection was from 49 to 33 patients (online supplementary table ST1f).

\section{Treatment patterns among patients remaining under follow-} up between 2015 and 2016

Among the 1451 patients from the 2015 active HCV cohort who remained in follow-up in 2016 (table 2), 154 $(10.6 \%)$ were on treatment or within 12 weeks of treatment completion in 2015, while a further 424 (29\%) patients commenced treatment during the study period. Treatment outcome was not yet known for 186 (12.8\%) 
Table 2 Treatment patterns and status in 2016

Treated or within 12 weeks of treatment completion between September 2015 and September 2016

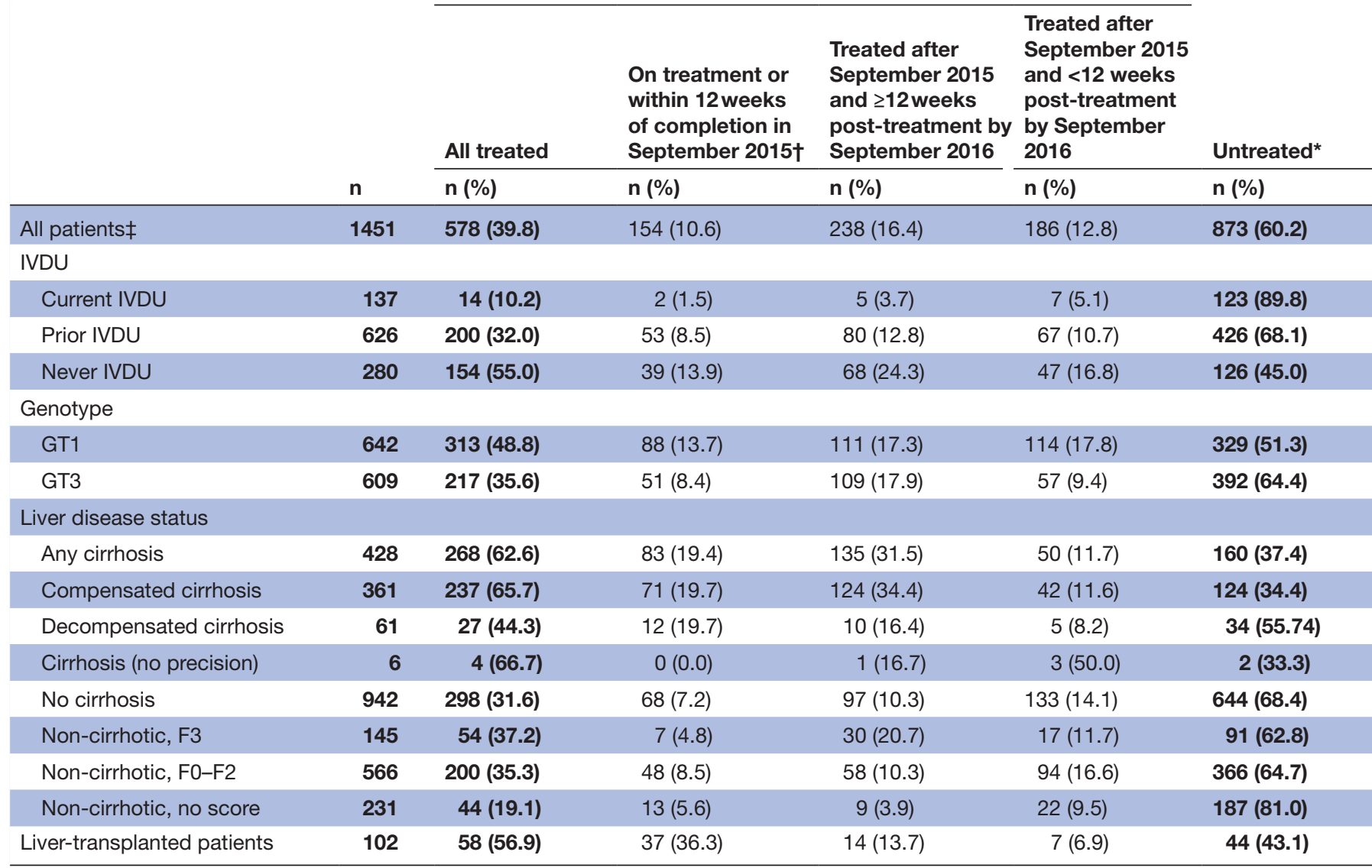

Data are presented as $\mathrm{n}(\%)$.

*Between September 2015 and September 2016.

†Did not receive any new treatment after September 2015.

$\ddagger$ All patients included in the 2015 active HCV cohort who had at least one visit since September 2014.

F0-F2, fibrosis stage 0-2; F3, fibrosis stage 3; GT1, genotype 1; GT3, genotype 3; IVDU, intravenous drug use.

patients at the end of the study period, as they had not yet completed their treatment or were less than 12 weeks post-treatment. Overall across the four study centres, $39.83 \%$ of patients from the 2015 active HCV cohort remaining in follow-up had been treated (with a known outcome) or were within 12 weeks post-treatment (treatment outcome not yet known) during the 1-year observation period.

The proportion of treated patients varied substantially according to cirrhosis status; $62.6 \%$ of patients with cirrhosis received HCV treatment during the study period, compared with only $31.6 \%$ of non-cirrhotic patients. IVDU status differed between treated and untreated patients; the proportion of the in-follow-up 2015 active HCV cohort who remained untreated between 2015 and 2016 was higher in patients with current (89.8\%) or prior $(68.1 \%)$ IVDU than those who never injected drugs $(45.0 \%)$. Regarding the cirrhosis status of patients with ongoing IVDU, in 2015 only $9.7 \%$ were cirrhotic and $75.8 \%$ were non-cirrhotic, with the remaining $14.5 \%$ non-specified due to omissions from patient notes. Thus, the trend for prioritising patients with cirrhosis for treatment was reflected in the lower proportion of treated IVDU patients. Finally, treatment status differed by HCV genotype, so that the proportion of untreated patients was higher among patients with GT3 (64.4\%) compared with GT1 $(51.3 \%) \mathrm{HCV}$.

Surprisingly, $55.7 \%$ of patients with decompensated cirrhosis remained untreated during the study period. All 34 patients still had decompensated cirrhosis in 2016: 1 of whom was on the transplant list, 13 (38.2\%) were alive, $2(5.9 \%)$ died from causes unrelated to HCV infection and $6(17.6 \%)$ from HCV-related causes; the status of $3(8.8 \%)$ patients was unknown, and 10 (29.4\%) patients had no new records during the subsequent follow-up, so that their outcomes could not be evaluated.

Regarding liver transplant status and treatment, more than half of 102 post-transplant patients (56.9\%) received at least one HCV treatment during the study period. 
Table 3 Outcomes in treated patients

\begin{tabular}{|c|c|c|c|c|}
\hline & All patients & $\begin{array}{l}\text { Patients with } \\
\text { decompensated } \\
\text { cirrhosis }\end{array}$ & $\begin{array}{l}\text { Patients } \\
\text { with compensated } \\
\text { cirrhosis }\end{array}$ & $\begin{array}{l}\text { Non-cirrhotic } \\
\text { patients }\end{array}$ \\
\hline & n (\%) & n (\%) & n (\%) & n (\%) \\
\hline Treated between 2015 and $2016^{*}$ & 383 & 22 & 191 & 160 \\
\hline \multicolumn{5}{|l|}{ Most recent treatment } \\
\hline $\mathrm{IFN} \pm \mathrm{RBV}$ & $20(5.2)$ & $0(0.0)$ & $2(1.1)$ & $18(11.2)$ \\
\hline IFN $\pm R B V+D A A$ & $44(11.5)$ & $0(0.0)$ & $11(5.8)$ & $33(20.6)$ \\
\hline DAA: simeprevir & $15(3.9)$ & $0(0.0)$ & $2(1.1)$ & $13(8.1)$ \\
\hline DAA: sofosbuvir & $29(7.6)$ & $0(0.0)$ & $9(4.7)$ & $20(12.5)$ \\
\hline IFN-free regimen & $316(82.5)$ & $22(100.0)$ & $178(93.2)$ & $107(66.9)$ \\
\hline Missing information & $2(0.5)$ & $0(0.0)$ & $0(0.0)$ & $1(0.6)$ \\
\hline Interruption due to adverse drug reaction & $6(1.6)$ & $1(4.6)$ & $0(0.0)$ & $5(3.1)$ \\
\hline Non-response & $3(0.8)$ & $1(4.6)$ & $0(0.0)$ & $2(1.3)$ \\
\hline Relapse & $8(2.1)$ & $2(9.1)$ & $3(1.6)$ & $3(1.9)$ \\
\hline SVR achieved & 357 (93.2) & $17(77.3)$ & $184(96.3)$ & 147 (91.9) \\
\hline Unknown & $5(1.3)$ & $0(0.0)$ & $2(1.1)$ & $2(1.3)$ \\
\hline
\end{tabular}

Data presented as $\mathrm{n}(\%)$.

*Includes patients treated prior to September 2015 whose treatment outcome was not yet known at the time (ie, they were $<12$ weeks from end of treatment).

DAA, direct-acting antiviral; HCV, hepatitis C virus; IFN, interferon; RBV, ribavirin; SVR, sustained virological response.

\section{Outcomes in treated patients}

Table 3 presents treatment received and its outcomes in the 383 patients from the 2015 active HCV cohort whose treatment outcome was known by 2016 (145 were already on treatment or within 12 weeks of completion in 2015 , after the exclusion of 9 patients with no visits between 2015 and 2016 and no available outcome data, and 238 were newly treated during the observation period).

Treatment regimens received varied substantially according to genotype and cirrhosis status. All 22 treated patients with decompensated cirrhosis received an IFN-free regimen and $17(77.3 \%)$ achieved SVR. One patient had no response to treatment, another interrupted treatment due to adverse drug reaction, two $(9.1 \%)$ patients relapsed, and one patient died of a cause unrelated to HCV infection.

Among the 191 treated patients with compensated cirrhosis, $98.9 \%$ of GT1 patients and $88.8 \%$ of GT3 patients received an IFN-free regimen, while $10.1 \%$ of GT3 patients were treated with $\mathrm{IFN} \pm$ ribavirin $(\mathrm{RBV})+\mathrm{so}-$ fosbuvir. Overall, 184 patients $(96.3 \%)$ with compensated cirrhosis achieved SVR by 2016 (95.5\% of GT1 and $96.6 \%$ of GT3), with $3(1.6 \%)$ patients experiencing a relapse, $2(1.1 \%)$ dying from causes unrelated to HCV infection and two (1.1\%) patients whose status was unknown.

A total of 160 non-cirrhotic patients were treated, of whom $56.3 \%$ and $33.6 \%$ were infected with GT1 and GT3
HCV, respectively. Most (74.4\%) GT1 patients received IFN-free regimens, compared with $51.9 \%$ of GT3 patients; $20.0 \%$ of GT1 and $25.9 \%$ of GT3 patients were treated with IFN \pm RBV $+\mathrm{DAA}$, while $3.3 \%$ of GT1 and $22.2 \%$ of GT3 patients were treated with $\mathrm{IFN} \pm \mathrm{RBV}$. The vast majority of non-cirrhotic patients $(91.9 \%)$ achieved SVR, including $93.3 \%$ of GT1 and $88.9 \%$ of GT3 patients. One patient with GT1 disease had a viral breakthrough, one patient with GT1 and one with GT3 infection did not achieve a response, while two GT1 $(2.2 \%)$ and one GT3 patients relapsed. Adverse drug reactions prompted discontinuations of treatment in five non-cirrhotic patients $(3.1 \%)$, of whom four $(7.41 \%)$ had GT3 HCV. All four of these patients received IFN-based regimens (three were treated with $\mathrm{IFN} \pm \mathrm{RBV}$ and one with $\mathrm{IFN} \pm \mathrm{RBV}+\mathrm{DAA}$ ).

\section{Patients lost to follow-up}

Of the 1605 patients in the 2015 active HCV cohort, 154 did not have a visit in the last 24 months prior to the 2016 index date, and as such were considered LTFU. In comparison with those patients in the 2015 cohort who were not LTFU, patients who were subsequently LTFU were typically younger ( 44.9 vs 49.4 years) and more likely to be affected by current IVDU (18.2\% vs $9.4 \%)$, non-cirrhotic $(76.6 \%$ vs $64.9 \%)$ and treatment-naïve $(76.6 \%$ vs $67.9 \%$ ) (table 4$)$. 
Open access

Table 4 Difference in characteristics in 2015 between patients who were LTFU versus not LTFU in 2016 2015 not LTFU

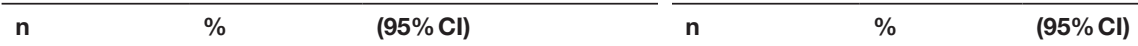

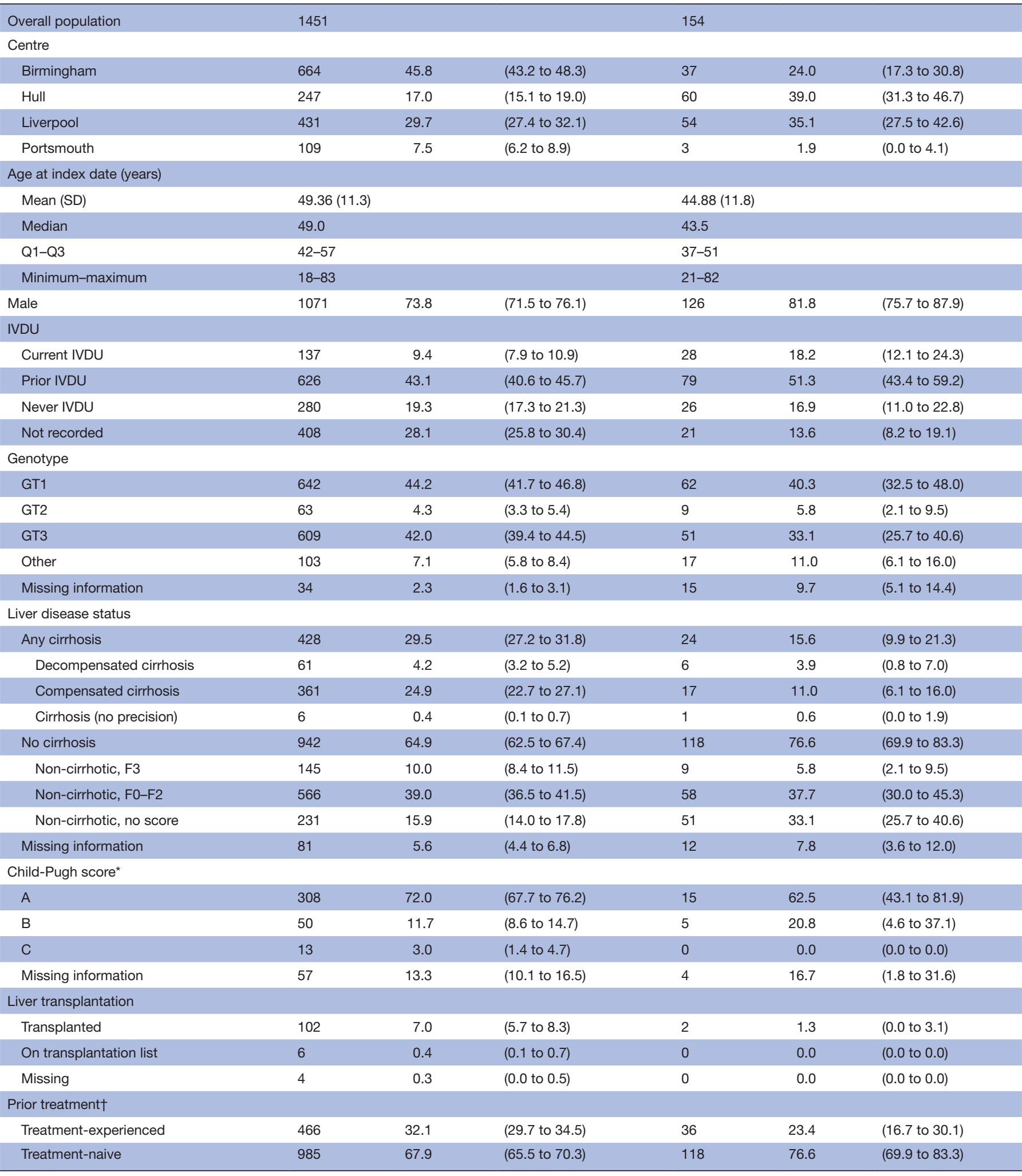

Continuous variables represented as mean. Categorical variables denoted as $\%(95 \% \mathrm{Cl})$.

*Includes only patients with cirrhosis.

tDoes not consider patients currently on treatment.

F0-F2, fibrosis stage 0-2; F3, fibrosis stage 3; GT1, genotype 1; GT2, genotype 2; GT3, genotype 3; IVDU, intravenous drug use; LTFU, lost to follow-up. 
LTFU patients were differentially distributed between study centres. At Portsmouth and Birmingham, the rate of loss to follow-up did not exceed 6\%. Conversely, high loss to follow-up rates were observed at Hull (60 of 307 LTFU patients, $19.6 \%$ ), attributable to the large prison population (explainable by greater levels of disengagement and probability of moving regions on release), and Liverpool (54 of 485 LTFU patients, 11.1\%). Hull was the largest contributor to the entire group of patients from the 2015 active HCV cohort who were LTFU, accounting for 60 of $154(39.0 \%)$ patients, followed by Liverpool (54 patients, $35.1 \%$ ).

\section{Newly referred patients}

Table 5 compares the baseline characteristics between the 337 patients who were newly referred to the study centres during the observation period and the 2015 active HCV cohort. Newly introduced patients, the majority of whom were enrolled at Hull, were on average younger than the 2015 active HCV cohort (45.1 vs 48.9 years) and more likely to be affected by current $(18.7 \%$ vs $10.3 \%)$ or historical (51.9\% vs $43.9 \%$ ) IVDU. In terms of HCV genotype distribution, they were more often infected with GT1 (48.7\% vs $43.9 \%)$ and less frequently with GT3 (32.3\% vs $41.1 \%)$ HCV. Newly referred patients were also more likely to be non-cirrhotic ( $76.9 \%$ vs $66.0 \%)$ than the 2015 active HCV cohort, and very few were liver transplant recipients $(0.6 \%$ vs $6.5 \%$ in the 2015 active HCV cohort).

\section{DISCUSSION}

The introduction of DAAs has been described as a 'step change' in the management and outcomes of HCV, ${ }^{18}$ representing the possibility of cure in most treated patients. ${ }^{5-7}$ Although early access programmes existed before June 2015, it was at about this time that DAAs were made available to patients with compensated cirrhosis treated in the National Health Service (NHS) ${ }^{15}$ although eligibility differed by genotype, extending to all GT1 patients but only those GT3 patients who had contraindications to IFN. ${ }^{15}$ From April 2016, access to DAAs was broadened to non-cirrhotic patients (but still prioritised to patients with cirrhosis). This staggered availability of DAA in different at-risk populations has meant that treatment has been prioritised to patients with the most severe forms of HCV-related liver disease. This history of DAA access policies is important in interpreting the composition and outcomes of the HCV cohorts in this study. The two index dates in our study were chosen to describe how the characteristics of patients with HCV remaining in contact with specialised care evolved with this changing landscape of treatment and related health policy, to provide important real-world data on the outcomes of antiviral treatment among a diverse patient cohort, representing a number of genotypes and liver disease stages. Overall, SVR rate in treated patients was $93.2 \%$, including 91.9\% in patients without liver cirrhosis and $96.3 \%$ in patients with compensated cirrhosis. However, despite all patients with decompensated cirrhosis receiving an IFN-free regimen (due to IFN being contraindicated in advanced liver disease), at $77.3 \%$, SVR rate was considerably lower among this difficult-to-treat patient group. The high SVR rates seen in our study are consistent with findings from other studies conducted in a range of countries, which often evaluated substantial proportions of patients with cirrhosis, or those who have failed prior treatment and/or had significant comorbidities, ${ }^{8-11}$ suggesting that excellent real-world outcomes of DAA treatment are reproducible across a range of, often challenging, patient populations.

Viral genotype and liver disease stage critically influenced the patient pathway. Treatment rate was more than double in patients with compensated cirrhosis compared with non-cirrhotic patients, although a progressive increase in the number of treated non-cirrhotic patients was observed over the study period. Furthermore, patients who were newly referred to the study centres during the observation period were more likely to be non-cirrhotic than patients from the prevalent 2015 active HCV cohort. These differences reflect the evolution of the recommendations regarding the use of the new DAAs during the study period, in England, with initial restriction of the use of DAAs for patients with severe liver damage. Of note, further treatments, including those for GT3-infected patients, became available on the NHS after the study period, so that therapy may have been deferred until their availability for some GT3 patients who would have received IFN during the period of our study. Treatment options availability could, therefore, explain the fewer GT3 than GT1 patients treated during the study period, and SVR was somewhat lower in patients infected with GT3 than GT1, most likely reflecting lower usage of IFN-free treatment in GT3 patients. Substantially more post-transplant patients with a GT1 infection were treated and removed from the active HCV cohort by 2016 compared with GT3-infected patients. Despite post-transplant patients being prioritised for treatment, due to their susceptibility to aggressive HCV progression in the setting of immunosuppression, only IFN-containing regimens were available to most GT3 patients at the time of our study. Also, the cohort of patients who were newly referred during the observation period included fewer GT3 patients than the prevalent 2015 active HCV cohort. Our results therefore suggest that a watch-and-wait approach was preferred in some GT3 patients pending the commissioning of an IFN-free regimen.

We have characterised patients who were LTFU during the observation period (ie, had no visits in the 24-month period prior to 2016), as these patients may be particularly difficult to engage and retain in care. It is, however, worth noting some LTFU patients may have moved, rather than disengaged with specialist care altogether, as we were unable to distinguish between these two situations. Patients who were LTFU were younger, more frequently male, more likely to be affected by current IVDU, have no cirrhosis and no prior treatment experience; the latter 
Open access

Table 5 Difference in characteristics between the 2015 active cohort and those newly introduced in 2016

2015 active HCV cohort 2016 newly introduced

\begin{tabular}{|c|c|c|c|c|c|c|}
\hline & & \\
\hline & $n$ & $\%$ & $(95 \% \mathrm{Cl})$ & $n$ & $\%$ & $(95 \% \mathrm{Cl})$ \\
\hline Overall population & 1605 & & & 337 & & \\
\hline \multicolumn{7}{|l|}{ Centre } \\
\hline Birmingham & 701 & 43.7 & (41.2 to 46.1$)$ & 36 & 10.7 & (7.4 to 14.0$)$ \\
\hline Hull & 307 & 19.1 & (17.2 to 21.1$)$ & 201 & 59.6 & (54.4 to 64.9 ) \\
\hline Liverpool & 485 & 30.2 & (28.0 to 32.5$)$ & 82 & 24.3 & (19.8 to 28.9$)$ \\
\hline Portsmouth & 112 & 7.0 & (5.7 to 8.2$)$ & 18 & 5.3 & (2.9 to 7.7$)$ \\
\hline \multicolumn{7}{|l|}{ Age at index date (years) } \\
\hline Mean (SD) & \multicolumn{3}{|l|}{$48.9( \pm 11.4)$} & \multicolumn{3}{|l|}{$45.1( \pm 10.7)$} \\
\hline Median & \multicolumn{3}{|l|}{49} & \multicolumn{3}{|l|}{44} \\
\hline Q1-Q3 & \multicolumn{3}{|l|}{$41-56$} & \multicolumn{3}{|l|}{$38-52$} \\
\hline Minimum-maximum & \multicolumn{3}{|l|}{$18-83$} & \multicolumn{3}{|l|}{$21-80$} \\
\hline Male & 1197 & 74.6 & (72.4 to 76.7 ) & 245 & 72.7 & (67.9 to 77.5$)$ \\
\hline \multicolumn{7}{|l|}{ IVDU } \\
\hline Current IVDU & 165 & 10.3 & (8.8 to 11.8$)$ & 63 & 18.7 & (14.5 to 22.9$)$ \\
\hline Prior IVDU & 705 & 43.9 & (41.5 to 46.4$)$ & 175 & 51.9 & (46.6 to 57.3 ) \\
\hline Never IVDU & 306 & 19.1 & (17.1 to 21.0$)$ & 68 & 20.2 & (15.9 to 24.5 ) \\
\hline Not recorded & 429 & 26.7 & (24.6 to 28.9 ) & 31 & 9.2 & (6.1 to 12.3$)$ \\
\hline \multicolumn{7}{|l|}{ Genotype } \\
\hline GT1 & 704 & 43.9 & (41.4 to 46.3$)$ & 164 & 48.7 & (43.3 to 54.0$)$ \\
\hline GT2 & 72 & 4.5 & (3.5 to 5.5$)$ & 21 & 6.2 & (3.7 to 8.8$)$ \\
\hline GT3 & 660 & 41.1 & (38.7 to 43.5$)$ & 109 & 32.3 & (27.3 to 37.3$)$ \\
\hline Other & 106 & 6.6 & (5.4 to 7.8$)$ & 24 & 7.1 & (4.4 to 9.9$)$ \\
\hline Missing information & 63 & 3.9 & (3.0 to 4.9$)$ & 19 & 5.6 & (3.2 to 8.1$)$ \\
\hline \multicolumn{7}{|l|}{ Liver disease status } \\
\hline Any cirrhosis & 452 & 28.2 & (23.4 to 33.0$)$ & 66 & 19.6 & (15.3 to 23.8$)$ \\
\hline Decompensated cirrhosis & 67 & 4.2 & (3.2 to 5.2$)$ & 18 & 5.3 & (2.9 to 7.7$)$ \\
\hline Compensated cirrhosis & 378 & 23.6 & (21.5 to 25.6$)$ & 47 & 13.9 & (10.2 to 17.6$)$ \\
\hline Cirrhosis (no precision) & 7 & 0.4 & (0.0 to 0.8$)$ & 1 & 0.3 & (0.0 to 0.9$)$ \\
\hline No cirrhosis & 1060 & 66.0 & (63.7 to 68.4$)$ & 259 & 76.9 & (72.4 to 81.4$)$ \\
\hline Non-cirrhotic, F3 & 154 & 9.6 & (8.2 to 11.0$)$ & 50 & 14.8 & (11.0 to 18.6$)$ \\
\hline Non-cirrhotic, F0-F2 & 624 & 38.9 & (36.5 to 41.3$)$ & 101 & 30.0 & (25.1 to 34.9$)$ \\
\hline Non-cirrhotic, no score & 282 & 17.6 & (15.7 to 19.4$)$ & 108 & 32.0 & (27.1 to 37.0$)$ \\
\hline Missing information & 93 & 5.8 & (4.7 to 6.9$)$ & 12 & 3.6 & (1.6 to 5.5$)$ \\
\hline \multicolumn{7}{|l|}{ Child-Pugh score* } \\
\hline A & 323 & 71.5 & (67.3 to 75.6$)$ & 33 & 50.0 & (37.9 to 62.1$)$ \\
\hline $\mathrm{B}$ & 55 & 12.2 & (9.2 to 15.2$)$ & 12 & 18.2 & (8.9 to 27.5$)$ \\
\hline C & 13 & 2.9 & $(0.0$ to 4.4$)$ & 9 & 13.6 & (0.0 to 21.9$)$ \\
\hline Missing information & 61 & 13.5 & (10.3 to 16.6$)$ & 11 & 16.7 & (7.7 to 25.7 ) \\
\hline \multicolumn{7}{|l|}{ Liver transplantation } \\
\hline Transplanted & 104 & 6.5 & (5.3 to 7.7$)$ & 2 & 0.6 & $(-0.2$ to 1.4$)$ \\
\hline On transplantation list & 6 & 0.4 & $(0.0$ to 0.7$)$ & 5 & 1.5 & (0.0 to 2.8$)$ \\
\hline Missing & 4 & 0.2 & $(0.0$ to 0.5$)$ & 1 & 0.3 & $(-0.3$ to 0.9$)$ \\
\hline \multicolumn{7}{|l|}{ Prior treatment $†$} \\
\hline Treatment-experienced & 502 & 31.3 & (29.0 to 33.5$)$ & 77 & 22.8 & (18.4 to 27.3 ) \\
\hline Treatment-naive & 1103 & 68.7 & (66.5 to 71.0$)$ & 260 & 77.2 & (72.7 to 81.6$)$ \\
\hline
\end{tabular}

Continuous variables represented as mean. Categorical variables denoted as $\%(95 \% \mathrm{Cl})$.

*Includes only patients with cirrhosis.

†Does not consider patients currently on treatment.

F0-F2, fibrosis stage 0-2; F3, fibrosis stage 3; GT1, genotype 1; GT2, genotype 2; GT3, genotype 3; HCV, hepatitis C virus; IVDU, intravenous drug use. 
may reflect a more recent HCV diagnosis or a lack of commitment to long-term treatment. Nonetheless, about one in six LTFU patients had cirrhosis, with the associated risk of progression to decompensated cirrhosis and a 5\%-30\% 5-year risk of developing liver cancer. ${ }^{2}$ Patients with current IVDU were not prioritised for treatment during the study period and $89.8 \%$ of them remained untreated, compared with just $45.0 \%$ of those with no IVDU history, so there was no specific incentive for IVDU patients to remain in care. Thus, keeping younger, male IVDU patients engaged with care appears a challenge, exemplified further by the substantial patient turnover at Hull, a centre serving a large prison population, where patients were more commonly younger and intravenous drug users than at the remaining centres. Nearly one in five patients from the active 2015 cohort enrolled at Hull were LTFU by 2016, while, conversely, the centre accounted for $59.6 \%$ of new referrals over the 1-year observation period. The likely explanation for this high turnover rate is that incarcerated patients with HCV are engaged with healthcare services, but do not continue with care at the Hull centre once released either because of disengagement or because of relocation to another geographical location following release. Future initiatives promoting engagement and treatment of young, male, IVDU patients, as well as patients released from prison appear of particular value to both individual patients and public health, as ongoing IVDU in the presence of an active HCV infection presents a high risk of disease spread, which may be reduced by effective treatment. Re-engaging these patients with healthcare services is a high priority, especially since LTFU patients before DAA treatment was widely available may not be aware of their present eligibility for IFN-free therapy.

While providing valuable information, our study has several limitations. First, we only included patients and collected information from selected centres. Thus, the generalisability of our findings should be considered against the study setting and the range of data collected; for instance, we may have been unable to fully capture the characteristics of patients difficult to engage with healthcare services, as some would not have been included in our study due to lack of contact with the study centres. Another limitation of our study relates to its relatively short (12 months) observation period, which did not fully span the evolution of HCV treatment in the UK from IFN-based to fully DAA-based. Consequently, the dynamic developments in the treatment of HCV in the last 2 years may mean that the characteristics of patients currently receiving treatment have changed from those described in our study. Finally, the retrospective nature of data collection could subject it to issues related to data access, completeness and accuracy, for example data entry errors.

Overall, our study demonstrated high cure rates achieved in UK patients with HCV as access to DAAs was progressively broadened during the observation period. However, there remains an unmet need for uniform access to DAAs among treatment-naïve patients with early stages of liver disease, and among disadvantaged and marginalised populations. Broadening DAA access and facilitating adherence to treatment may ultimately achieve HCV elimination and, consequently, removing the substantial burden that this disease poses on patients and healthcare services.

\section{Author affiliations}

${ }^{1}$ Bristol-Myers Squibb, Uxbridge, UK

${ }^{2}$ Health Economics and Outcomes Research, Cardiff, UK

${ }^{3}$ Bristol-Myers Squibb Pharmaceuticals, Uxbridge, UK

${ }^{4}$ Institute of Infection and Global Health, University of Liverpool, Liverpool, UK ${ }^{5}$ Gastroenterology Department, Portsmouth Hospitals NHS Trust, Portsmouth, UK ${ }^{6}$ Gastroenterology Department, Hull and East Yorkshire Hospitals NHS Trust, Hull, UK ${ }^{7}$ Liver and Hepatobiliary Unit, University Hospitals Birmingham NHS Foundation Trust, Birmingham, UK

Acknowledgements We thank Ryan Miller of HEOR for assistance with data analyses within the study, Dr Karolina Badora and Dr Daniel Sugrue, both of HEOR, for assistance with developing the manuscript, Hosnieh Fathi for her contributions to the study design, and the staff at the four hospital sites for data collection.

Contributors LL, DM, NRH, MH and JG conceptualised and designed the study. AMG, RA, LC, YG-D, LM and DM were responsible for data collection. MH and JG were responsible for data analysis. All authors contributed to the interpretation of the results, preparation and review of the manuscript, and approval of the final manuscript for publication.

Funding This work was supported by Bristol-Myers Squibb, which provided support for the data analysis and medical writing for this study. The funding agreement ensured the authors' independence in designing the study, interpreting the data and preparing the manuscript for publication.

Competing interests MH and JG are employees of HEOR. NRH and LL are employees of BMS. LC and YG-D have previously received research funding from AbbVie Pharmaceuticals relevant to HCV. AMG has received consultancy honoraria from Janssen and Cepheid, has been employed by Roche Pharma Research \& Early Development (pRED) (no stocks, shares or patents) and has acted as the principal investigator on research grants alongside Janssen, ViiV, BMS and Gilead. RA has served as a speaker or advisory board member for AbbVie Pharmaceuticals, Falk Pharma, Gilead, Intercept, Norgine and Novartis. LM has no conflict of interest. DM has accepted honoraria and has acted as adviser to BMS, Gilead Sciences and AbbVie.

\section{Patient consent for publication Not required.}

Ethics approval Ethical approval was obtained prior to any data collection in each centre, and the data set was anonymised within each centre (as a function within the eCRF) prior to sending to the contract research organisation (CRO) for validation and analysis of the combined data set from all four centres.

Provenance and peer review Not commissioned; externally peer reviewed.

Data sharing statement № additional data are available.

Open access This is an open access article distributed in accordance with the Creative Commons Attribution Non Commercial (CC BY-NC 4.0) license, which permits others to distribute, remix, adapt, build upon this work non-commercially, and license their derivative works on different terms, provided the original work is properly cited, appropriate credit is given, any changes made indicated, and the use is non-commercial. See: http://creativecommons.org/licenses/by-nc/4.0/.

\section{REFERENCES}

1. World Health Organization. Hepatitis C. 2017 http://www.who.int/ mediacentre/factsheets/fs164/en/ (Accessed 3 Apr 2018).

2. El-Serag HB. Epidemiology of viral hepatitis and hepatocellular carcinoma. Gastroenterology 2012;142:1264-73.

3. Public Health England. Hepatitis C in the UK 2017 report. 2017 https://www.gov.uk/government/uploads/system/uploads/ attachment_data/file/632465/HCV_in_the_uk_report_2017.pdf (Accessed 3 April 2018). 
4. Public Health England. Hepatitis C in England 2018 report. 2018 https://www.gov.uk/government/uploads/system/uploads/ attachment_data/file/693917/HCV_in_England_2018.pdf (Accessed 3 Apr 2018).

5. Bourlière M, Gordon SC, Flamm SL, et al. Sofosbuvir, velpatasvir, and voxilaprevir for previously treated HCV infection. N Engl J Med 2017;376:2134-46.

6. Jacobson IM, Lawitz E, Gane EJ, et al. Efficacy of 8 weeks of sofosbuvir, velpatasvir, and voxilaprevir in patients with chronic HCV infection: 2 phase 3 randomized trials. Gastroenterology 2017;153:113-22.

7. Falade-Nwulia O, Suarez-Cuervo C, Nelson DR, et al. Oral directacting agent therapy for hepatitis $C$ virus infection: a systematic review. Ann Intern Med 2017;166:637-48.

8. Aspinall Al, Shaheen AA, Kochaksaraei GS, et al. Real-world treatment of hepatitis $\mathrm{C}$ with second-generation direct-acting antivirals: initial results from a multicentre Canadian retrospective cohort of diverse patients. CMAJ Open 2018;6:E12-E18.

9. Bachofner J, Valli PV, Bergamin I, et al. Excellent outcome of direct antiviral treatment for chronic hepatitis C in Switzerland. Swiss Med Wkly 2018;148:w14560.

10. Conti F, Brillanti S, Buonfiglioli F, et al. Safety and efficacy of direct-acting antivirals for the treatment of chronic hepatitis $\mathrm{C}$ in a real-world population aged 65 years and older. $J$ Viral Hepat 2017;24:454-63.

11. Su F, Beste LA, Green PK, et al. Direct-acting antivirals are effective for chronic hepatitis $C$ treatment in elderly patients: a real-world study of 17487 patients. Eur J Gastroenterol Hepatol 2017;29:686-93.
12. Yek $\mathrm{C}$, de la Flor $\mathrm{C}$, Marshall $\mathrm{J}$, et al. Effectiveness of direct-acting antiviral therapy for hepatitis $C$ in difficult-to-treat patients in a safety-net health system: a retrospective cohort study. BMC Med 2017; 15:204.

13. European Association for the Study of the Liver. Electronic address: easloffice@easloffice.eu. EASL Recommendations on Treatment of Hepatitis C 2016. J Hepatol 2017;66:153-94.

14. McEwan P, Kim R, Yuan Y. Assessing the cost utility of responseguided therapy in patients with chronic hepatitis $\mathrm{C}$ genotype 1 in the UK using the MONARCH model. Appl Health Econ Health Policy 2013;11:53-63.

15. NHS England. Clinical Commissioning Policy Statement: Treatment of chronic Hepatitis C in patients with cirrhosis. 2015 https://www. england.nhs.uk/commissioning/wp-content/uploads/sites/12/2015/ 06/hep-c-cirrhosis-polcy-statmnt-0615.pdf (Accessed 9 Apr 2018).

16. Public Health England. Hepatitis Ci n the UK. 2018 https://assets. publishing.service.gov.uk/government/uploads/system/uploads/ attachment data/file/732469/HCV IN THE UK 2018 UK.pdf (Accessed 29 Apr 2019).

17. Royal College of General Practitioners. Guidance for the prevention, testing, treatment and management of hepatitis $C$ in primary care. 2017 http://www.hepctrust.org.uk/sites/default/files/PreventionTesting-Treatment-and-management-of-hep-C-in-primary-care \% 281\%29.pdf (Accessed 29 Apr 2019).

18. Public Health England. Hepatitis C in the UK. 2016 report. 2016 https://assets.publishing.service.gov.uk/government/uploads/ system/uploads/attachment_data/file/565459/Hepatitis_C_in_the _ UK_2016_report.pdf (Accessed 12 Apr 2018). 